\title{
Optical properties of high-quality InGaAs/InAlAs multiple quantum wells
}

\author{
S. Gupta, P. K. Bhattacharya, J. Pamulapati, and G. Mourou \\ Ultrafast Science Laboratory and Solid State Electronics Laboratory, Department of Electrical Engineering \\ and Computer Science, University of Michigan, Ann Arbor, Michigan 48109
}

(Received 12 September 1990; accepted for publication 6 December 1990)

\begin{abstract}
We have measured the narrowest half-width at half-maximum photoluminescence linewidth of $2.8 \mathrm{meV}$, in 40 -period lattice-matched $\operatorname{In}_{0.53} \mathrm{Ga}_{0.47} \mathrm{As} / \mathrm{In}_{0.52} \mathrm{Al} \mathrm{l}_{0.48} \mathrm{As}$ multiple quantum wells, grown by molecular-beam epitaxy with growth interruption. A simple analysis of the linewidth suggests that the structure has near perfect interfaces. Temperature-dependent photoluminescence linewidth data indicate impurity incorporation due to the growth interruption. However, the high quality of the multiple quantum well is not impaired as is seen in the room-temperature absorption data, where excitonic features up to $n=3$ sublevel are clearly seen. Carrier lifetime in this multiple-quantum-well system has been measured, we believe for the first time, using the picosecond photoluminescence correlation technique. A lifetime of $860 \mathrm{ps}$ is obtained, which is similar to the value obtained for high-quality $\mathrm{GaAs} / \mathrm{AlGaAs}$ and $\mathrm{In}_{0.53} \mathrm{Ga}_{0.47} \mathrm{As} / \mathrm{InP}$ quantum wells. This further confirms the high quality obtained in this ternary material system using growth interruption.
\end{abstract}

\section{INTRODUCTION}

$\mathrm{In}_{0.53} \mathrm{Ga}_{0.47} \mathrm{As}$ and $\mathrm{In}_{0.53} \mathrm{Ga}_{0.47} \mathrm{As} / \mathrm{In}_{0.52} \mathrm{Al}_{0.48} \mathrm{As}$ multiple quantum wells (MQWs) lattice matched to InP have received a lot of attention for their application to optoelectronic devices for optical-fiber communication. By changing the MQW parameters it is possible to change the emission or absorption energy in the wavelength range of 1.0$1.67 \mu \mathrm{m}$, which covers the regions of low loss and low dispersion in silica optical fibers. This multiple quantum well is grown entirely with a single anion species, which makes control of growth parameters and resulting interface quality easier compared to the more common $\mathrm{In}_{0.53} \mathrm{Ga}_{0.47} \mathrm{As} / \mathrm{InP}$ system. This multiple-quantum-well system is being used in lasers, ${ }^{1,2}$ detectors, ${ }^{3,4}$ and electroabsorption/electro-optic modulators. ${ }^{5,6}$ In these devices the structural quality of the interfaces and optical properties such as the absorption coefficient, photoluminescence (PL) intensity, and linewidth can play an important role. These properties are, in turn, intimately related to the growth techniques and parameters, source purity, and intrinsic material properties.

Other than some early photoluminescence and absorption studies, ${ }^{7,8}$ there is no detailed report on the optical properties of the InGaAs/InAlAs/InP MQW system. An interesting and important feature of this InP-based system is that by increasing or decreasing the amount of In in the InGaAs (well) material from the lattice-matched composition, the well region can be placed under biaxial compressive or tensile strain, respectively. Such strain dramatically alters the valence-band structure and thereby the optical properties. ${ }^{9}$ In this paper, however, we will confine ourselves to studies on the lattice-matched $\mathrm{In}_{0.33} \mathrm{Ga}_{0.47} \mathrm{As} /$ $\operatorname{In}_{0.52} \mathrm{Al}_{0.48} \mathrm{As}$ multiple quantum well.

We have grown a series of $\mathrm{In}_{0.53} \mathrm{Ga}_{0.47} \mathrm{As} /$ $\mathrm{In}_{0.52} \mathrm{Al}_{0.48} \mathrm{As} \mathrm{MQWs}$ under well-controlled molecularbeam-epitaxial (MBE) conditions, to be described in the next section. We have made variable-temperature photoluminescence and absorption measurements. The half-width at half-maximum (HWHM) linewidth of the exciton peak in the low-temperature PL is $2.8 \mathrm{meV}$, which, to our knowledge, is the lowest achieved in this MQW system. We have analyzed temperature-dependent linewidth data to obtain the homogeneous and inhomogeneous components of the linewidth and exciton lifetimes. Roomtemperature absorption spectra clearly show excitonic transitions up to $n=3$, which also confirms the high quality of the samples. Finally, we have measured the lifetime of carriers in MQWs by picosecond time-resolved photoluminescence using the correlation spectroscopy technique. ${ }^{10,11}$ We find that the carrier lifetime in the MQW region is $\sim 860$ ps. In what follows, MBE growth is described in Sec. II, the measurement techniques are outlined in Sec. III, the results are presented and discussed in Sec. IV, and finally the results are summarized in Sec. V.

\section{MOLECULAR-BEAM-EPITAXIAL GROWTH}

$p^{+}-i(\mathrm{MQW})-n^{+}$samples were grown on Sn-doped $n^{+}$-InP substrates (Crystacomm) in a Varian Gen II MBE growth facility. The diodes are schematically shown in Fig. 1. The system preparation and growth is briefly described. The effusion cells without the crucibles are baked at $1400^{\circ} \mathrm{C}$ for $10 \mathrm{~h}$. Clean PBN crucibles are next inserted into the cells and are baked at temperatures ranging from 1300 to $1500^{\circ} \mathrm{C}$ for $11 \mathrm{~h}$. The growth charges consisting of $8 \mathrm{~N}$ Ga (Alusuisse), 7N As (JohnsonMatthey), $6 \mathrm{~N} \mathrm{Al}$ (Cominco), 7N In (Johnson-Matthey), and the dopant species $\mathrm{Si}$ and $\mathrm{Be}$ are loaded into the individual cells, and the growth chamber is evacuated to $\sim 10^{-9}$ Torr. It may be noted that before charging the In, it is baked for periods ranging from 24 to $48 \mathrm{~h}$ at $700{ }^{\circ} \mathrm{C}$ in an ultrapure $\mathrm{H}_{2}$ ambient. The chamber is then externally baked at $220^{\circ} \mathrm{C}$ for 5 days. After removal of the baking shrouds and subsequent cooling, the vacuum in the chamber is usually in the range $(2-4) \times 10^{-11}$ Torr. The source materials are then baked for $6-7 \mathrm{~h}$ at $100-200^{\circ} \mathrm{C}$ above their normal operating temperatures. The exception is As, 


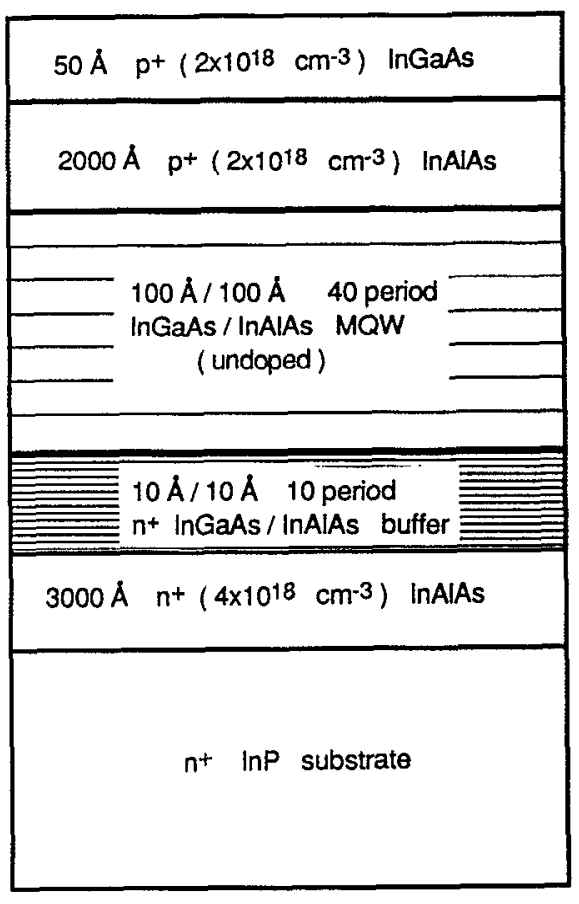

FIG. 1. Schematic of the $p-i$ (MQW)- $n$ diodes grown by molecular-beam epitaxy.

which is baked at $40^{\circ} \mathrm{C}$ above the operating temperature for $4 \mathrm{~h}$. We find that the steps outlined above are crucial for the growth of InGaAs and InAlAs with desirable electrical and optical properties. A thick AlGaAs/GaAs layer is usually grown first in a newly prepared system. Several calibration growth runs are made to adjust alloy composition and growth rate, and optimize the transport properties and radiative efficiency. In our growth system undoped $\operatorname{In}_{0.53} \mathrm{Ga}_{0.47} \mathrm{As}$ is usually $n$ type with $N_{D}-N_{A}=(0.9-3.0) \times 10^{15} \mathrm{~cm}^{-3}, \mu_{n}(300 \mathrm{~K})$ $=10500 \mathrm{~cm}^{2} /(\mathrm{V} \mathrm{s})$, and $\mu_{n}(77 \mathrm{~K})=40000-50000$ $\mathrm{cm}^{2} /(\mathrm{V} \mathrm{s}) . \mathrm{In}_{0.52} \mathrm{Al}_{0.48} \mathrm{As}$ grown under normal conditions has high resistivity.

The 40-period 100- $\AA / 100-\AA \mathrm{MQW}$ samples as shown in Fig. 1 were grown at a rate of $0.7 \mu \mathrm{m} / \mathrm{h}$ and at a temperature of $520^{\circ} \mathrm{C}$, as read by an infrared pyrometer. Prior to growth the (001) InP substrate was heated under an As flux to $540^{\circ} \mathrm{C}$ until a clear $(2 \times 4)$ As-stabilized reconstruction was seen in the reflection high-energy electrondiffraction (RHEED) pattern. Growth commenced with a $0.3-\mu \mathrm{m}$-thick Si-doped $\left(4 \times 10^{18} \mathrm{~cm}^{-3}\right)$ $n^{+}-\mathrm{In}_{0.52} \mathrm{Al}_{0.48} \mathrm{As}$ layer, followed by ten periods of Sidoped InGaAs $(10 \AA) / \operatorname{InAlAs}(10 \AA)$ superlattice. This superlattice serves two purposes: It smoothens the growth front after growing the buffer InAlAs layer since the migration rate of $\mathrm{Ga}$ on surface is much larger than $\mathrm{Al}$, and it also acts as a dislocation filter. The exact mechanism of this filtering is still largely unknown, ${ }^{12}$ but it is believed that propagating threading dislocations are filtered and are prevented from reaching the active region. Forty periods of the MQW and the doped layers following it are then grown under identical conditions. It should be noted that to ob- tain high-quality interfaces, growth was interrupted after each InAlAs layer in the MQW. Although this improves the interface quality, it also leads to increased impurity incorporation, and hence an intermediate value of 2-min interruption time was chosen. Following this MQW region, a $0.2-\mu \mathrm{m}$-thick Be-doped $\left(2 \times 10^{18} \mathrm{~cm}^{-3}\right)$ $p^{+}-\operatorname{In}_{0.52} \mathrm{Al}_{0.48}$ As layer was grown, capped by a 50 - $\AA$-thick $p^{+}$-InGaAs layer for achieving ohmic contacts. The V/III beam pressure ratio was 47 for the growth of $\mathrm{In}_{0.53} \mathrm{Ga}_{0.47} \mathrm{As}$ and 51 for the growth of $\operatorname{In}_{0.52} \mathrm{Al}_{0.48} \mathrm{As}$. Single-crystal $\mathrm{x}$-ray measurements confirmed that the lattice mismatch $\Delta a / a_{0}$ in the MQW was less than $10^{-4}$.

\section{MEASUREMENT TECHNIQUES}

\section{A. Photoluminescence and absorption measurements}

Temperature-dependent PL and absorption measurements were made with a 1-m Jarrell-Ash scanning spectrometer. The sample is placed in a variable-temperature liquid-He cold-finger cryostat with temperature control from 8 to $300 \mathrm{~K}$. For the PL measurements, dc excitation is provided by a $6328-\AA$ He-Ne laser, whereas for subpicosecond excitation a balanced colliding pulse modelocked (CPM) dye laser is used. The luminescence is detected by a liquid- $\mathrm{N}_{2}$-cooled $\mathrm{Ge}$ detector. A tungsten lamp is used for the absorption measurements. The absorption measurements were further corrected for the lamp mission spectrum and the detector responsivity curve.

\section{B. Picosecond-time-correlated photoluminescence spectroscopy}

For high-speed optoelectronic device applications, it is important to measure and know the carrier lifetimes. Depending on the type of device and its mode of operation, the temporal response is determined by a combination of radiative recombination, tunneling, trapping, scattering, and diffusion. The rate of these processes can be measured in suitably designed structures by observing the PL decay in the picosecond time scale with a sensitive and fast photodetector. A convenient arrangement is the streak camera. Unfortunately, this camera is not sensitive in the wavelength range coinciding with the transition energies in InGaAs/InAlAs MQWs.

An alternative technique is the picosecond photoluminescence correlation spectroscopy (PCS) technique, ${ }^{10.11}$ which gives the correlation of the carrier population if a nonbimolecular rate process (e.g., trapping, tunneling, etc.) also occurs in parallel with the radiative recombination process. Two advantages of this technique are than an ultrafast detector is not needed, and therefore it can be applied to any wavelength range. The time resolution of this technique is limited only by the laser pulsewidth of the excitation source. Hence, picosecond time resolution is easily achieved.

The experimental schematic of the PCS technique is shown in Fig. 2. The intensity of luminescence from photogenerated carrier recombination in a semiconductor is proportional to the product of the population of the 
Balanced CPM dye laser

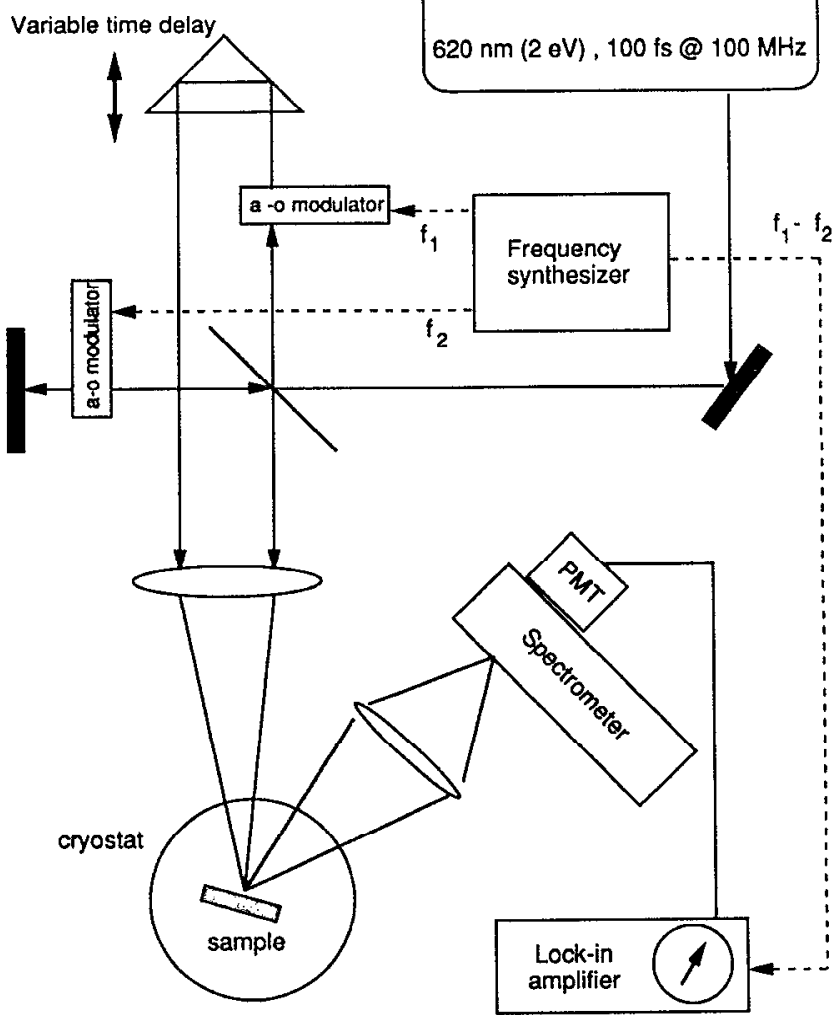

FIG. 2. Experimental schematic of the time-resolved photoluminescence correlation spectroscopy setup.

conduction-band electron density and the valence-band hole density. In the correlation scheme, two optical pulses are used, with a controllable delay $\tau$ between them. The first pulse creates an electron and hole population $n_{1}(t)$ and $p_{1}(t)$, respectively. The second pulse generates $n_{2}(t+\tau)$ and $p_{2}(t+\tau)$. The cross correlation of the two populations is obtained by measuring the nonlinear luminescence signal by chopping the two beams at two different frequencies $\left(f_{1}, f_{2}\right)$ and measuring the desired signal at the sum or difference frequency. ${ }^{10}$ This correlation signal $I(\tau)$ produced due to these two pulses can be written as

$$
I(\tau) \propto \int\left[n_{1}(t) p_{2}(t+\tau)+n_{2}(t+\tau) p_{1}(t)\right] d t .
$$

In our measurements the optical excitation consists of $100-$ fs pulses (100-MHz repetition rate) at $620 \mathrm{~nm}(2.0$ eV) from a balanced colliding pulse mode-locked (CPM) laser. In the InGaAs/InAlAs MQW carriers are therefore generated throughout the absorption region. The photoluminescence is collected and dispersed by a $\frac{1}{4}-\mathrm{m}$ Jarrell-Ash spectrometer. The luminescence is detected by a liquid- $\mathrm{N}_{2-}$ cooled $\mathrm{Ge}$ detector. The ring configuration of the CPM laser gives two synchronized beams. A computercontrolled delay is introduced in one of the beams. Both beams are modulated by acousto-optic (AO) modulators, which are driven by two outputs of a dual-channel frequency synthesizer in the frequency range of a few $\mathrm{MHz}$,

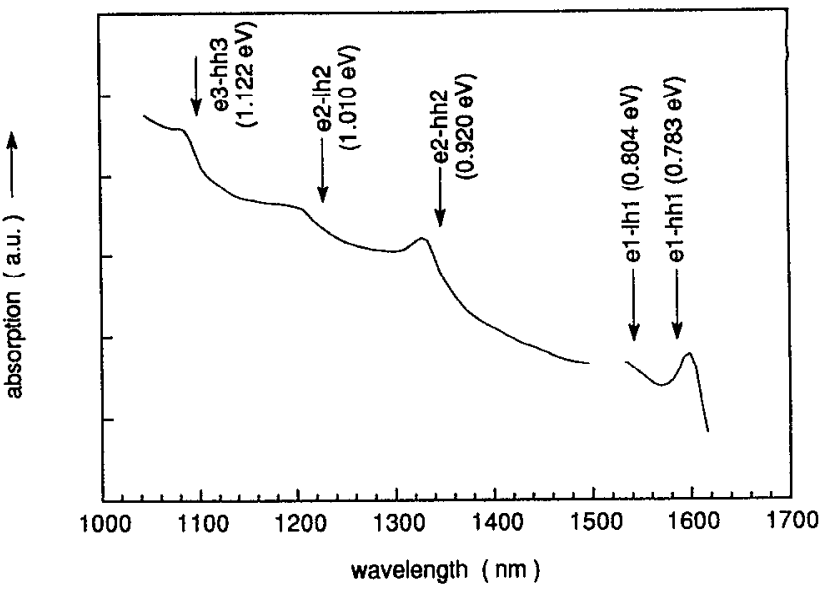

(a)

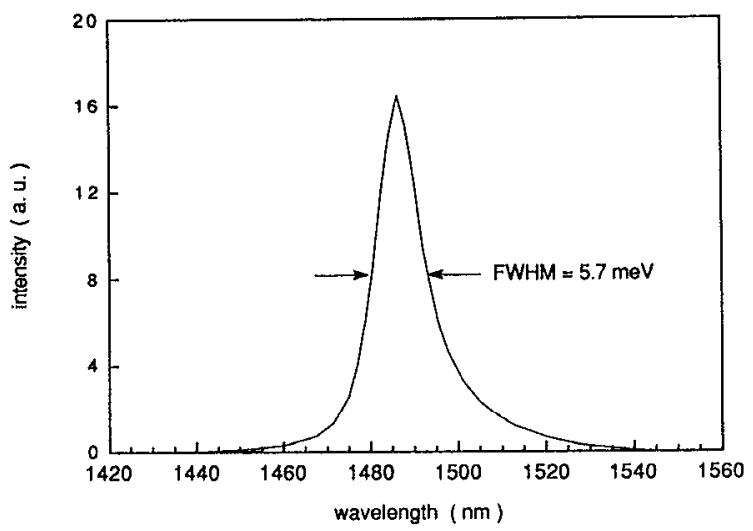

(b)

FIG. 3. (a) Absorption spectra of $\operatorname{In}_{0.53} \mathrm{Ga}_{0.47} \mathrm{As} / \mathrm{In}_{0.52} \mathrm{Al} \mathrm{l}_{0.48} \mathrm{As} \mathrm{MQW}$ at room temperatuie showing up to $n-3$ cxcitonic transitions. The calculated transitions based on a finite-square-well model with $\Delta E_{c}$ $=60 \% \Delta E_{g}$ are marked by arrows: (b) low temperature $(T=8 \mathrm{~K})$ photoluminescence measurement of the MQW showing a FWHM linewidth of $5.7 \mathrm{meV}$.

and separated in frequency by $1-50 \mathrm{kHz}$, depending on the response speed of the detector. Lock-in detection was used at the difference frequency generated in a mixer using the inputs from the frequency synthesizer. Since the CPM laser has a $1 / f$ noise spectrum with the corner frequency at approximately few hundred $\mathrm{kHz}$, this high-frequency modulation, especially of the difference, results in vastly superior signal-to-noise ratio as compared to $1-2-\mathrm{kHz}$ modulation using mechanical choppers. Unfortunately, the lownoise Ge detector we have used for this measurement is a large-area detector and has a factory-preset high gain, thereby restricting its $3-\mathrm{dB}$ response to about $1 \mathrm{kHz}$. Hence, the above-mentioned advantages of $\mathrm{AO}$ modulation could not be fully exploited.

\section{RESULTS AND DISCUSSION}

\section{A. Absorption and photoluminescence}

The measured room-temperature absorption spectra at zero bias of the MQW sample of Fig. 1 is shown in Fig. 3 (a). The spectra contains excitonic features up to the 
subband energy level at $n=3$. Both heavy- and light-hole resonances are seen at $n=1$ (or the ground state) and the next energy subband of $n=2$, as well as the $n=3$ heavyhole resonance. The measured transitions compare very well with the calculated values based on a finite-squarewell model using $\Delta E_{c}=60 \%$ of $\Delta E_{g}$ for the InGaAs/ InAlAs material system as shown in Fig. 3. The data suggest a sample quality comparable to the best GaAs/ AlGaAs MQW reported in literature. ${ }^{13}$ At low temperatures $\sim 8 \mathrm{~K}$, the various features in the absorption spectra sharpen along with the temperature-dependent shift in the energies toward the higher-energy side.

The low-temperature $(T=8 \mathrm{~K})$ photoluminescence of the MQW sample is shown in Fig. 3(b). The main peak is at the lowest energy of $0.834 \mathrm{eV}$. We believe that this peak originates from heavy-hole free-exciton transitions $e_{1}$ $\mathrm{hh}_{1}$. A small tail towards the lower-energy side is presumably from impurity transitions because of their increased incorporation during growth interruption. This will be discussed more in the next section. Much weaker peaks are seen at higher energies. In order of their relative intensities (peak height) they are a broad peak at $1.508 \mathrm{eV}$, which originates from the doped $\operatorname{In}_{0.52} \mathrm{Al}_{0.48}$ As layers, a peak at $1.105 \mathrm{eV}$, which probably originates from the superlattice buffer layer, and under picosecond excitation, another peak at $0.96 \mathrm{eV}$, which we believe originates from the higherorder $e_{2}$-hh $h_{2}$ transition.

The linewidth [full width at half maximum (FWHM)] of the main $e_{1}-\mathrm{hh}_{1}$ peak in the $8-\mathrm{K}$ spectrum is $5.7 \mathrm{meV}$, which we believe is the narrowest being reported until date. This further confirms the high quality of the quantum wells. The linewidth of the excitonic transitions is determined by the InGaAs alloy quality, the InAlAs alloy quality, and intrawell (interface roughness) and interwell size fluctuations. The linewidth due to alloy broadening is given by: ${ }^{14}$

$$
\sigma_{a}=\sqrt{\sigma_{1}^{2}+\sigma_{2}^{2}}
$$

where

$$
\begin{aligned}
& \sigma_{1}=\sigma_{B 1}^{a} \frac{\Delta_{\mathrm{QW}}}{\Delta_{B 1}} \frac{1}{\sqrt{P_{0}^{\mathrm{ex}}}}, \\
& \sigma_{2}=\sigma_{B 2}^{a} \frac{\Delta_{\mathrm{QW}}}{\Delta_{B 2}} \frac{1}{\sqrt{1-P_{0}^{\mathrm{ex}}}} .
\end{aligned}
$$

Here $\sigma_{B 1}^{a}$ and $\sigma_{B 2}^{a}$ represent the PL linewidth in the InAlAs and InGaAs, respectively. $\Delta_{\mathrm{QW}}$ represents the variation of the electron-hole subband energy difference with variation in the alloy composition, and $\Delta_{B}$ 's represent the variation in the band gap of the material with alloy composition. $P_{0}^{\mathrm{cx}}$ is the exciton fraction in the barrier. In addition to $\sigma_{a}$, the fluctuations due to the interface roughness also affect the lincwidth. The interface roughness can be described by two-dimensional islands (or valleys) of height $\delta_{1}$ and lateral radius $\delta_{2}$. The linewidth is dependent upon both $\delta_{1}$ and $\delta_{2}$. Its dependence on $\delta_{2}$ is much more complicated. If $\delta_{2} \sim R_{\mathrm{ex}}$ (where $R_{\mathrm{ex}}$ is the exciton radius), the linewidth is given by

$$
\sigma_{\mathrm{IR}}=\left.\delta_{1} \frac{\partial E^{e h}}{\partial W}\right|_{W_{0}},
$$

where $E^{\text {eh }}=$ separation between the electron and hole subbands, $W=$ well size, and $W_{0}=$ mean well size of the quantum well.

In the case of a MQW, an additional broadening arises due to the variation in $W_{0}$ from well to well. The linewidth limited by this fluctuation is given by an expression similar to Eq. (3).

The dependence of the excitonic linewidth on alloy broadening and interface roughness in the InGaAs/ InAlAs MQW has been calculated by Singh and Bajaj. ${ }^{14}$ In order to interpret the PL linewidth measured in this study, we need to know the values of $\sigma_{B 1}$ and $\sigma_{B 2}$. The best values of excitonic linewidths measured in InAlAs and InGaAs are $8-10$ and $1.5-3.0 \mathrm{meV}$, respectively. The linewidth in InGaAs represents that for a perfect alloy, while the larger linewidth in InAlAs has been associated with some clustering present in the system. ${ }^{15}$ For a $100-\AA$ quantum well, due to the high barrier discontinuities for the InAlAs/ InGaAs quantum well, only a very small fraction of the exciton $(\sim 3 \%)$ penetrates and sees the alloy clustering in the InAlAs region. From Eqs. (1)-(3) the linewidth contribution due to the alloy quality of the structure is given by $\sigma_{a} \sim 2.5 \mathrm{meV}$. We therefore estimate that the contribution of the interface roughness is about $3-4 \mathrm{meV}$ to the total linewidth. We note that the linewidth due to a one monolayer fluctuation at the surface with a lateral extension of the fluctuation which is equal to the exciton radius is $\sim 3.5 \mathrm{meV}$ for a $100-\AA$ InAlAs/InGaAs quantum well. Based on our calculations, we conclude that the interface roughness at the InAlAs/InGaAs interface is described by two-dimensional islands which are one monolayer high $(\sim 2.8 \AA)$ with $\sim 100$ - $\AA$ radius laterally. This implies that the normal interface of the quantum well (InAlAs on InGaAs), which from kinetic considerations is smoother than the inverted interface (InGaAs on InAlAs), has a roughness of the order of a monolayer or less. The inverted interface has, at best, roughness of the order of a monolayer. Furthermore, there is negligible interwell size fluctuations.

\section{B. Temperature-dependent luminescence measurements}

Extensive studies have been made to characterize the low- and room-temperature excitonic properties in GaAs/ AlGaAs quantum wells. Very little work has been done with the InGaAs/InAlAs quantum-well system. The first analysis of excitonic absorption and PL data at low and room temperature in this QW system was reported by Wciner et al. ${ }^{7}$ and by us. ${ }^{16}$ Excitonic linewidths, both homogeneous and inhomogeneous, are of critical importance in the performance of modulation devices based on quantum wells. The linewidth controls the contrast ratio of optical modulators as well as the efficiency of self electrooptic switching devices proposed by Miller et al. ${ }^{17}$ 


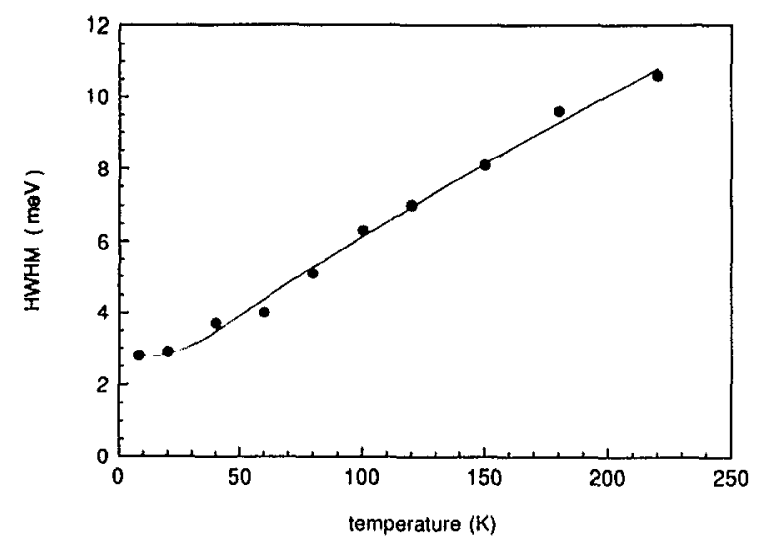

FIG. 4. Temperature dependence of the photoluminescence linewidth of the MQW sample (dots). The measured data is fitted to Eq. (4) (solid line), which also includes the impurity broadening. The fitting parameters are $\Gamma_{\text {Inhum }}=2.8 \mathrm{meV}, \Gamma_{\mathrm{op}}=12.6 \mathrm{meV}, \Gamma_{\text {Imp }}=8.3 \mathrm{meV}$, and $\left\langle E_{b}\right\rangle$ $=8.6 \mathrm{meV}$.

We have made PL measurements on the InGaAs/ InAlAs MQW samples in the temperature range 8-230 K with the system described in Sec. III. In analyzing the linewidth data, our interest focused on the heavy-hole excitonic transition which is dominant all through the temperature range. The low-energy side of the dominant heavy-hole transition overlaps with impurity boundexciton transitions at low temperatures, while the highertemperature spectra consist of both heavy- and light-hole luminescence peaks, with the light-hole transitions few tens of meVs away on the high-energy sides. Thus the highenergy sides were selected for curve fitting below $70 \mathrm{~K}$, which is the critical temperature for the total disappearance of the impurity bound excitons and the occurrence of the light-hole transitions. Above $70 \mathrm{~K}$ the low-energy sides were chosen for the analysis to avoid the interference of the light-hole transitions.

The temperature dependence of the heavy-hole photoluminescence linewidth (HWHM) is shown in Fig. 4. The above-mentioned factors lead to an inhomogeneous component of broadening which is temperature independent. As the temperature increases, due to the increasing phonon population, scattering by longitudinal optical (LO) phonons becomes dominant. This gives rise to the homogeneous component of the linewidth broadening. An additional inhomogeneous broadening arises due to the scattering of the exciton by impurity donors or acceptors. The total contribution to the PL HWHM can therefore be written as the sum of these three factors:

$$
\Gamma=\Gamma_{\text {inhom }}+\frac{\Gamma_{o p}}{h \omega_{\mathrm{LO}} / e^{k T}-1}+\Gamma_{\mathrm{imp}} e^{\left\langle E_{b}\right\rangle / k T},
$$

where $\omega_{L O}=$ longitudinal optical phonon frequency, $\left\langle E_{b}\right\rangle=$ average binding energy of impurity, $\Gamma$ 's are the respective linewidth constants.

At the lowest measured temperature of $8 \mathrm{~K}$, the PL $\mathrm{HWHM}=2.8 \mathrm{meV}$ was chosen as the $\Gamma_{\text {inhom }}$ component, as all other factors are not important, and from the experimental data, the HWHM value also seems to reach this minima. A value of $h \omega_{\mathrm{LO}}=33 \mathrm{meV}$ was used. ${ }^{18}$ As was mentioned earlier, the growth interruption leads to an increased impurity incorporation. The tail on the low-energy side of the PL in Fig. 3(b) probably arises due to these impurity bound transitions which are not ionized at this low temperature. If this broadening mechanism is ignored, the above expression fits the measured data with unreasonably large values for $\Gamma_{O p}$. Including the impurity broadening and assuming an impurity level with an average binding energy of $\sim 8.6 \mathrm{meV}$, the obtained fit is excellent as shown in Fig. 4. Exciton lifetimes are calculated from the homogeneous linewidths using the uncertainty principle. From the value of $\Gamma_{0 p}$, at room temperature, the mean exciton ionization time is about $150 \mathrm{fs}$.

\section{Carrier lifetime in the MQW system}

So far, we have discussed only the time-independent properties of this material system. Considering the relativcly greater difficulty involved in the growth of the InAlAs/InGaAs system, it is important to note that a quality of the interface structure as predicted by theory has been obtained. This should therefore be reflected in the dynamic properties of the carriers in the multiple quantum well. In particular, the carrier lifetimes should be about the same as those expected in any high-quality MQW, like in the GaAs/AlGaAs system. That is, due to the high quality of the interface, no additional trapping/recombination centers at the normal or inverted interface, as well as in the well region, should be present. It is to be noted that the carrier lifetime in the quantum wells would be different from that in the bulk material because of the enhanced recombination rate. In order to determine the carrier lifetime in the quantum well, the technique of photoluminescence correlation spectroscopy (PCS) is used. As has been mentioned earlier in Sec. III, the small fraction of trapping processes which are inevitably present in an MBE-grown material help in generating the photoluminescence correlation signal.

Figures 5(b) and 5(c) show the measured PCS signal at the two wavelengths corresponding to the energies of 0.834 and $1.508 \mathrm{eV}$. Note that the actual correlation signal is symmetrical about $\tau=0$. Here, only the part corresponding to positive delays $\tau$ is shown. As mentioned earlier, the lower energy corresponds to the $e_{1}-\mathrm{hh}_{1}$ transition in the MQW and the broader one at $1.508 \mathrm{eV}$ corresponds to the band-to-band transition in the $p^{+}$-InAlAs layer (Fig. 1). Referring to Fig. 5(a), following the subpicosecond carricr excitation by the laser pulse, carriers are predominantly generated in the top absorbing $p^{+}$-InAlAs layer. The average optical power of $2 \mathrm{~mW}$ per beam, focused to an $\sim 100-\mu$ m-diam spot, corresponds to a photon flux of about $10^{12}$ photons $/ \mathrm{cm}^{2}$ per pulse. A small part of these recombine in the InAlAs region, and the remainder diffuse to and are captured by the quantum wells, which explains the very fast decay of the $1.508-\mathrm{eV}$ luminescence as observed in Fig. 5(b). This capture process would therefore give rise to a delayed rise in the time-resolved luminescence of the $n=1 \mathrm{MQW}$ transition and is manifested by the rounded top in the correlation measurement of Fig. 


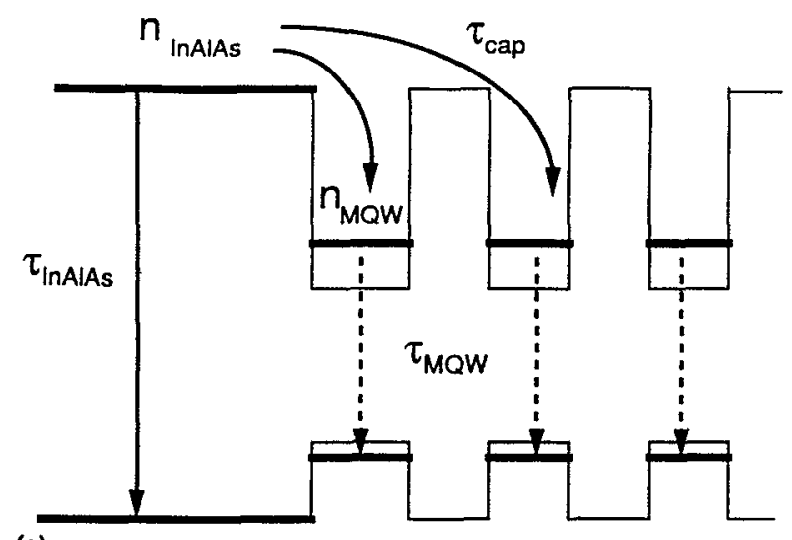

(a)
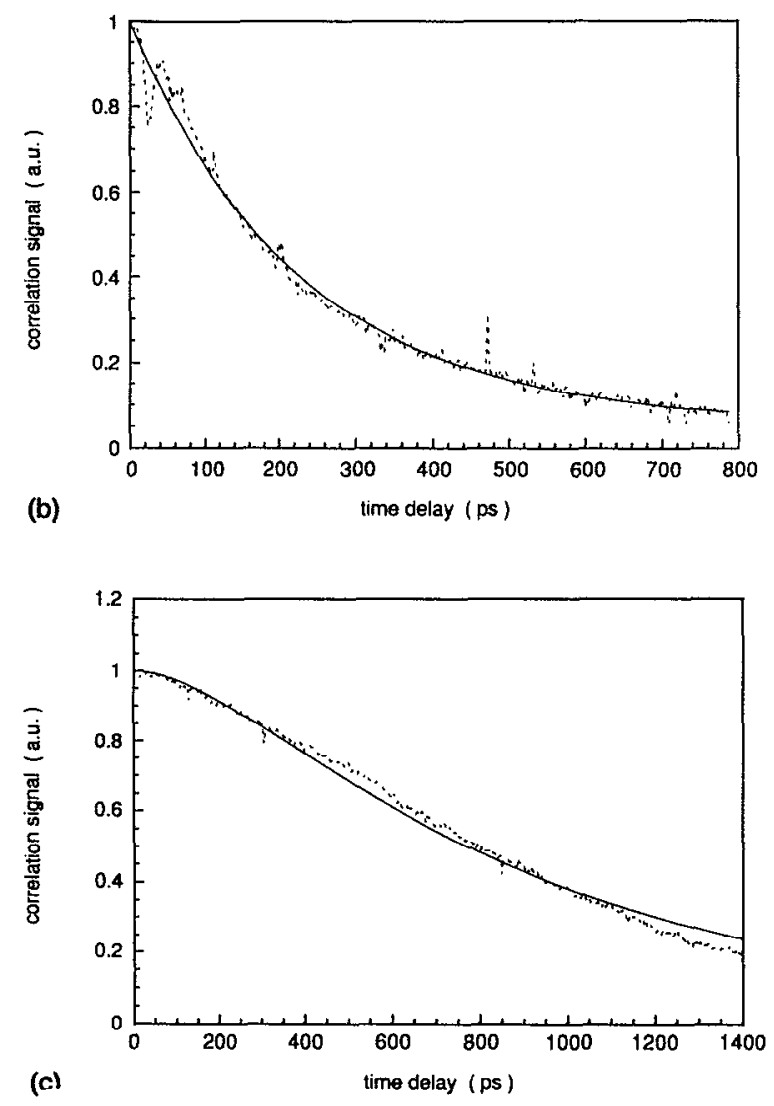

FIG. 5. (a) Schematic of the rate processes in the time-resolved photoluminescence. (b) Photoluminescence correlation signal (dashed line) for the InAlAs band edge ( $1.508 \mathrm{eV})$, showing the exponential decay. The data are fit to the correlation of the solution of Eq. (5) (solid line) with a single time constant of $\tau=\left(1 / \tau_{\text {InAlAs }}+1 / \tau_{\text {cap }}\right)^{-1}=228 \mathrm{ps.} \mathrm{(c)} \mathrm{Pho-}$ toluminescence correlation signal corresponding to the $n=1$ excitonic transition $(0.834 \mathrm{eV})$ in the MQW (dashed line). The data is fit to the correlation of the solution obtained from Eq. (6) (solid line), with the parameters $\tau_{\text {cap }}=290 \mathrm{ps}$ and $\tau_{\mathrm{MQW}}=860 \mathrm{ps}$.

$5(c)$. The overall decay of the MQW luminescence is due to the finite carrier lifetime in the MQW.

The experimental results can be analyzed quantitatively on the basis of a simple two-level rate equation model, suggested by the schematic in Fig. 5(a), to extract the carrier-capture time and lifetime in the MQW system. We assume that the electron and hole populations remain close as the initial photoinjected carrier density is assumed to be much larger than the density of traps. One can therefore write down the following rate equations:

$$
\begin{aligned}
& \frac{d n_{\text {InAlAs }}}{d t}=G(t)-\frac{n_{\text {InAlAs }}}{\tau_{\text {cap }}}-\frac{n_{\text {InAlAs }}}{\tau_{\text {InAlAs }}}, \\
& \frac{d n_{\mathrm{MQW}}}{d t}=\frac{n_{\text {InAlAs }}}{\tau_{\text {cap }}}-\frac{n_{\mathrm{MQW}}}{\tau_{\mathrm{MQW}}} .
\end{aligned}
$$

Here, $n_{\text {InAlAs }}$ and $n_{\mathrm{MQW}}$ represent the populations of the InAlAs band and the $n=1 \mathrm{MQW}$ sublevel, respectively. $G(t)$ represents the carrier generation function, which for our laser pulse can be treated as a $\delta$ function. $\tau_{\text {InAlAs }}$ and $\tau_{\mathrm{MQW}}$ represent the effective carrier lifetimes in the InAlAs region and the $n=1$ sublevel of the MQW, respectively. $\tau_{\text {cap }}$ represents the capture time of the photogenerated carriers by the quantum well, which includes the diffusion of carriers from the absorbed region to the MQW region and their subsequent relaxation to the $n=1$ sublevel.

The above equations can be solved analytically, and their autocorrelation function can be computed and fitted to the measured data. Note that Eq. (5) has an exponentially decaying solution, and therefore its correlation is also the same exponential. This is shown in Fig. 5(b). The best fit to the data is obtained for the total decay rate of (1/ $\left.\tau_{\text {InAlAs }}+1 / \tau_{\text {cap }}\right)^{-1}=228$ ps. Solving Eq. (6) and taking its correlation to fit the data in Fig. 5(c), one gets $\tau_{\text {cap }}=290 \mathrm{ps}$ and $\tau_{\mathrm{MQW}}=860 \mathrm{ps}$. Thus $\tau_{\text {InAlAs }} \sim 1.1 \mathrm{~ns}$ is obtained. To the best of our knowledge, this represents the first direct measurement of the carrier lifetime in this MQW system. This value of $\tau_{\mathrm{MQW}}=860 \mathrm{ps}$ is very close to the value typically obtained for the GaAs/AlGaAs MQW system ${ }^{19}$ and in the $\mathrm{In}_{0.53} \mathrm{Ga}_{0.47} \mathrm{As} / \mathrm{InP}$ quantum-well system. ${ }^{20}$ This further implies that the well/barrier material and the interfaces (normal and inverted) in the InGaAs/ InAlAs system, similar to those in the GaAs/AlGaAs system, can be obtained. Also, the increased impurity incorporation due to the growth interruption has negligible effect on the carrier lifetime in the MQW.

\section{CONCLUSIONS}

We have measured dc and time-resolved optical properties of a high-quality lattice-matched InGaAs/InAlAs MQW system grown by MBE. The narrowest photoluminescence linewidth ever reported, of $2.8 \mathrm{meV}$ (HWHM), has been measured at a temperature of $8 \mathrm{~K}$. Roomtemperature absorption clearly show excitonic features up to the $n=3$ sublevel. From a simple analysis of this, less than one monolayer interface roughness for the normal interface and at most a one monolayer roughness for the inverted interface can be deduced. This indicates that very close to the theoretical best interface quality can be achieved in this material system. Temperature-dependent photoluminescence data indicate presence of impurities due to the use of growth interruption. The carrier lifetime in the MQW has also been measured and shows a value similar to that in high-quality GaAs/AlGaAs samples. Thus, the increased impurity incorporation due to growth 
interruption does not seem to adversely affect the carrier lifetime in the MQW. In conclusion, it is possible to grow a very-high-quality lattice-matched InGaAs/InAlAs MQW system with very good interfaces and carrier lifetimes.

\section{ACKNOWLEDGMENTS}

The authors thank Professor J. Singh for several helpful discussions. The work was supported by the Office of Naval Research under Grant No. N00014-90-J-1831, and by the Air Force Office of Scientific Research (University Research Initiative) under Contract No. AFOSR-90-0214.

${ }^{1}$ K. Alavi, H. Temkin, W, R. Wagner, and A. Y. Cho, Appl. Phys. Lett. 42, 254 (1983).

${ }^{2}$ H. Temkin, K. Alavi, W. R. Wagner, T. P. Pearsall, and A. Y. Cho, Appl. Phys. Lett. 42, 845 (1983).

${ }^{3}$ H. T. Griem, S. Ray, J. L. Freeman, and D. L. West, Appl. Phys. Lett. 56, 1067 (1990).

${ }^{4}$ C. Y. Chen, Y. M. Pang, P. A. Garbinski, A. Y. Cho, and K. Alavi, Appl. Phys. Lett. 43, 308 (1983).

${ }^{5} \mathrm{~K}$. Wakita, O. Mitomi, I. Kotaka, S. Nojima, and Y. Kawamura, IEEE Photon. Technol. Lett. 1, 441 (1989).

${ }^{\circ}$ S. A. Pappert, R. J. Orazi, T. T. Vu, S. C. Lin, A. R. Clawson, and P.
K. L. Yu, IEEE Photon. Technol. Lett. 2. 257 (1990).

${ }^{7}$ J. S. Weiner, D. S. Chemla, D. A. B. Miller, T. H. Wood, D. Sivco, and A. Y. Cho, Appl. Phys. Lett. 46, 619 (1985).

${ }^{8}$ F-Y. Juang, P. K. Bhattacharya, and J. Singh, Appl. Phys. Lett. 48, 290 (1986).

${ }^{9}$ S. C. Hong, G. P. Kothiyal, N. Debbar, P. K. Bhattacharya, and J. Singh, Phys. Rev. B 37, 878 (1988).

${ }^{10}$ D. Rosen, A. G. Doukas, Y. Budansky, A. Katz, and R. R. Alfano, Appl. Phys. Lett. 39, 935 (1981).

"M. B. Johnson, T. C. McGill, and A. T. Hunter, J. Appl. Phys. 63, 2077 (1988).

${ }^{12}$ B. W. Dodson, J. Electron. Mater. 19, 503 (1990).

${ }^{13}$ R. Dingle and W. Wiegmann, J. Appl. Phys. 46, 4312 (1975).

${ }^{14}$ J. Singh and K. K. Bajaj, J. Appl. Phys. 57, 5433 (1985).

${ }^{15}$ W. P. Hong, P. K. Bhattacharya, and J. Singh, Appl. Phys. Lett. 50, 618 (1987)

${ }^{16}$ Y. Chen, G. P. Kothiyal, J. Singh, and P. K. Bhattacharya, Superlatt. Microstruct. 3, 657 (1987).

${ }^{17}$ D. A. B. Miller, D. S. Chemla, T. C. Damen, T. H. Wood, C. A. Burrus, A. C. Gossard, and W. Wiegmann, IEEE J. Quantum Electron. QE-21, 1462 (1985).

${ }^{18}$ A. Pinczuk, J. M. Worlock, R. E. Nahory, and M. A. Pollack, Appl. Phys. Lett. 33, 461 (1978).

${ }^{19}$ E. O. Gobel, H. Jung, J. Kuhl, and K. Ploog, Phys. Rev. Lett. 51, 1588 (1983).

${ }^{20}$ U. Cebulla, G. Bacher, A. Forchel, G. Mayer, and W. T. Tsang, Phys. Rev. B 39, 6257 (1989). 